\section{$\underset{\substack{\text { hommes } \\ \text { \& migrations }}}{ }$}

\section{Hommes \& migrations}

Revue française de référence sur les dynamiques

migratoires

1317-1318 | 2017

L'Europe en mouvement

\title{
Les migrations italiennes dans la France contemporaine
}

Les nouveaux visages d'une mobilité européenne historique

Hadrien Dubucs, Thomas Pfirsch, Ettore Recchi et Camille Schmoll

\section{(2) OpenEdition}

1 Journals

\section{Édition électronique}

URL : http://journals.openedition.org/hommesmigrations/3871

DOI : 10.4000/hommesmigrations.3871

ISSN : 2262-3353

Éditeur

Musée national de l'histoire de l'immigration

\section{Édition imprimée}

Date de publication : 1 avril 2017

Pagination : 59-67

ISBN : 978-2-919040-38-4

ISSN : 1142-852X

Référence électronique

Hadrien Dubucs, Thomas Pfirsch, Ettore Recchi et Camille Schmoll, « Les migrations italiennes dans la France contemporaine », Hommes \& migrations [En ligne], 1317-1318 | 2017, mis en ligne le 01 avril 2020, consulté le 04 janvier 2020. URL : http://journals.openedition.org/hommesmigrations/3871 ; DOI : 10.4000/hommesmigrations.3871 


\section{LES MIGRATIONS ITALIENNES DANS LA FRANCE CONTEMPORAINE LES NOUVEAUX VISAGES D'UNE MOBILITÉ EUROPÉENNE HISTORIQUE}

Par HADRIEN DUBUCS, ENeC, université Paris-Sorbonne, THOMAS PFIRSCH, UMR Géographie-Cités, université de Valenciennes, ETTORE RECCHI, Sciences Po, Observatoire sociologique du changement, CNRS, et CAMILLE SCHMOLL, Géographie-Cités, Institut universitaire de France, université Paris Diderot.

Comme d'autres pays européens, la France connaît depuis une vingtaine d'années une reprise de l'immigration italienne qui demeure encore mal connue. Statistiques et enquêtes de terrain permettent de montrer les différences de ce nouveau flux avec les anciennes vagues migratoires italiennes : des effectifs plus faibles, des parcours plus métropolitains et une part importante des jeunes actifs qualifiés. Plus que la crise économique et la recherche d'un travail, la mobilité des jeunes diplômés de la classe moyenne répond à des blocages structurels de leur société d'origine, incapable de renouvellement générationnel. Elle est révélatrice des formes migratoires émergentes d'une nouvelle génération d'Européens mobiles.

L'immigration italienne en France renvoie à une histoire longue et reflète bien les recompositions économiques, sociales et culturelles qui ont affecté les mobilités intra-européennes dans la période contemporaine. Cette histoire fait l'objet d'une considérable littérature scientifique tant en France qu'en Italie ${ }^{1}$ et a suscité une abondante production artistique qui lui confère une place de choix dans l'imaginaire collectif français relatif aux migrations. On connaît moins bien, en revanche, les formes les plus récentes de cette migration, alors que les débats sont vifs sur une éventuelle reprise de l'émigration italienne - et plus largement sudeuropéenne - depuis les années $2000^{2}$. Les effets de l'intégration européenne sur ces « nouvelles migrations » restent peu étudiés : internationalisa- 
tion des cursus universitaires et des compétences professionnelles, densification des infrastructures transfrontalières de transport et accélération des déplacements, renforcement des cadres réglementaires et des dispositifs européens de la mobilité (Schengen, Erasmus...). Enfin, on connaît mal l'impact sur ces migrations de la crise économique qui a touché l'Italie à la fin des années 2000.

À partir d'une analyse de la

Ainsi, entre 1990 et 2005,

les entrées officielles d'Italiens

en France oscillent autour

de 3000 par an, mais on

observe ensuite une explosion

dans le sillage de la crise

économique. Les entrées

doublent entre 2010 et 2015 ,

passant de 3784 à 9020 littérature et d'une série d'enquêtes menées depuis 2013, cet article entend éclairer les formes contemporaines des migrations italiennes en France, et plus particulièrement à Paris. Il met en débat l'idée d'une continuité historique par rapport aux vagues migratoires antérieures et se demande en quoi cette nouvelle migration italienne est révélatrice des formes contemporaines des mobilités européennes. Dans quelle mesure peut-on parler d'une nouvelle vague migratoire italienne en France? Quels sont les visages de cette migration ? Est-on face à une nouvelle génération de migrants italiens et, plus largement, d'Européens mobiles?

\section{Une nouvelle vague migratoire italienne en France?}

Comme elle a lieu à l'intérieur de l'espace Schengen, la migration italienne en France laisse peu de traces dans les statistiques officielles, ce qui implique de croiser les sources d'information en combinant le traitement de données statistiques et la réalisation d'enquêtes de terrain. L'article repose sur trois sources principales. Une enquête par entretiens approfondis a d'abord été menée à Paris à partir de novembre 2012 auprès de jeunes actifs Italiens récemment arrivés dans la capitale. Fondée sur une approche biographique, elle fournit des informations détaillées sur les représentations, les circulations, les réseaux familiaux, les pratiques urbaines, les liens avec le pays d'origine des nouveaux migrants italiens ${ }^{3}$. Deuxièmement, un questionnaire en ligne a été diffusé en 2015 à partir de groupes Facebook et de mailing lists d'Italiens résidant à Paris. Les 515 enquêtés ont fourni des informations sur leur parcours personnel et professionnel, leur situation résidentielle, leurs relations avec l'Italie ou encore des données rarement collectées telles que le niveau de revenu net $^{4}$. Enfin, un cadrage général de ces enquêtes a été effectué grâce au croisement de statistiques officielles françaises et italiennes.

Que nous apprennent les chiffres officiels sur l'immigration italienne actuelle en France? Depuis 2003, les Italiens ne sont plus soumis à l'obtention d'un permis de séjour et ne sont donc plus enregistrés chaque année par le ministère de l'Intérieur. Côté français, les seules sources permettant de mesurer leur présence sur le territoire sont donc les enquêtes de recensement. On y voit une baisse constante du «stock » d'Italiens en France depuis 1980, ce dernier passant de 340000 en 1982 à 173000 en 2009. Mais cette baisse reflète surtout le vieillissement et la dilution des anciennes vagues migratoires italiennes dans la société française au gré des naturalisations. En 2009, près de $65 \%$ des immigrés italiens recensés par l'Institut national de la statistique et des études économiques (Insee) ont plus de 60 ans.

Pour avoir une idée de l'ampleur de la nouvelle immigration transalpine, il faut se tourner vers les données italiennes qui, grâce au système des registres de populations, permettent une mesure des flux annuels entre les deux pays. Les Italiens ont en effet l'obligation légale de s'enregistrer auprès de leur consulat après six mois de résidence à l'étranger et, même si seule une minorité 
d'entre eux le font réellement, ces chiffres donnent un ordre de grandeur des flux migratoires annuels. Ainsi, entre 1990 et 2005, les entrées officielles d'Italiens en France oscillent autour de 3000 par an, mais on observe ensuite une explosion dans le sillage de la crise économique. Les entrées doublent entre 2010 et 2015, passant de 3784 à $9020^{5}$. Ce phénomène s'inscrit dans un contexte plus général de reprise de l'émigration italienne depuis le début des années 2000, essentiellement tournée vers l'Union européenne. La tendance s'est encore accentuée avec la crise économique avec un accroissement très net depuis 2010 de l'émigration vers la France, mais aussi l'Allemagne (+197\%), le Royaume-Uni (+156 \%) et la Suisse $(+140 \%)^{6}$. Depuis 2005, la France est systématiquement la quatrième destination de cette nouvelle vague migratoire italienne.

Pour autant, ces migrations restent très en deçà de l'ère de l'immigration de masse et elles s'inscrivent dans un mouvement d'augmentation générale de l'immigration européenne en France depuis le début des années 2000. En effet, les arrivées d'Italiens ne sont pas plus importantes que celles en provenance des autres grands voisins de l'hexagone. Selon l'Insee, en 2014, les Italiens représentaient seulement $4 \%$ des immigrés entrés en France en 2012, tout comme les Allemands (4\%), les Britanniques (5 \%) ou les Espagnols (5 \%). Dans le sillage de l'élargissement de l'UE en 2004, cette augmentation de l'immigration européenne en France a d'abord concerné les pays d'Europe de l'Est mais, depuis la crise de l'euro, les flux en provenance des pays d'Europe du Sud ont le plus augmenté?.

La spécificité de l'immigration italienne actuelle réside donc moins dans son ampleur que dans sa diversité sociale (retraités, cadres expatriés, étudiants...), et surtout dans la place qu'y occupent de plus en plus les jeunes actifs diplômés.

\section{De nouvelles manières de migrer}

Les nouveaux migrants italiens en France se distinguent nettement de leurs prédécesseurs des anciennes vagues migratoires, souvent peu qualifiés, issus des campagnes pauvres de la péninsule et arrivés en France en suivant parents et compaesani. Les entretiens menés à Paris rendent bien compte d'un sentiment de réel décalage par rapport aux immigrés plus anciennement arrivés dans la capitale :

«Si tu veux rencontrer une autre typologie d'Italiens, il faut aller le matin tôt faire la queue au consulat et là tu vas rencontrer tous les vieux Italiens, les papis de 70 ans qui habitent plutôt la banlieue est de Paris et qui ont créé à lépoque des associations régionales, du genre: "Les gens des Pouilles à Paris". » (Antonio, cadre supérieur du privé, 35 ans).

Il s'agit aujourd'hui d'une migration plus individuelle. Elle concerne à égale proportion des femmes ou des hommes arrivés en France sans s'appuyer sur des filières familiales et régionales, mais ayant eu fréquemment recours à des réseaux universitaires euroLes nouveaux migrants italiens en France se distinguent nettement de leurs prédécesseurs des anciennes vagues migratoires, souvent peu qualifiés, issus des campagnes pauvres de la péninsule et arrivés en France en suivant parents et compaesani. péens. En 2011, 49 \% des migrants italiens arrivés en France au cours des cinq années précédentes étaient des femmes ${ }^{8}$.

Les nouveaux migrants italiens se distinguent également de leurs prédécesseurs par leur origine géographique, désormais essentiellement urbaine et septentrionale. En 2014, on comptait 17 émigrations pour 10000 résidents dans les régions du Nord-Ouest de l'Italie, 16 dans le Nord-Est, et seule- 
ment 13 dans le Centre et 12 dans le Sud ${ }^{9}$. Les nouveaux migrants italiens proviennent donc majoritairement des régions les plus riches et urbanisées de la péninsule, et qui comptent déjà la plus forte concentration de capital humain ${ }^{10}$. L'émigration semble être encouragée, par un effet de compétition-entraînement-émulation, au sein des lieux les plus dynamiques de léconomie italienne. La sousreprésentation du Mezzogiorno parmi les régions de départ s'explique aussi par la reprise de l'émigration interne en Italie, les régions du Nord constituant un bassin d'accueil pour les Méridionaux, quitte à ce que ceux-ci reprennent ensuite la route de l'international pour une deuxième émigration ${ }^{11}$.

Les entretiens menés auprès des jeunes migrants italiens à Paris montrent que, si ces derniers ont parfois des traditions migratoires dans leurs familles, et ont pu être socialisés précocement à la mobilité par les lycées linguistiques ou les voyages dans l'enfance, aucun ne compte de parents en France ou n'a « suivi » un membre de sa famille ou de sa région pour migrer. Certains l'ont même fait contre l'avis

Une autre grande différence

entre ces nouveaux migrants

italiens et les anciennes

vagues migratoires est leur niveau élevé de qualification.

D'après les enquêtes annuelles de recensement, les Italiens

sont, avec les Espagnols,

les plus qualifiés des migrants récents en France. sa formation d'ingénieur à l'École centrale grâce au programme européen Time et qui a rencontré sa future femme française dans un camp d'été au Portugal rassemblant de jeunes européens, se décrit comme un «pur produit de l'Europe » et a le sentiment d'appartenir à une génération " pionnière » dans son aisance à envisager une carrière hors d'Italie (neuf des onze camarades italiens de sa promotion travaillent aujourd'hui hors d'Italie).

\section{Des migrants italiens jeunes, actifs et diplômés}

Une autre grande différence entre ces nouveaux migrants italiens et les anciennes vagues migratoires est leur niveau élevé de qualification. D'après les enquêtes annuelles de recensement, les Italiens sont, avec les Espagnols, les plus qualifiés des migrants récents en France. En 2011, $56 \%$ des Italiens et $59 \%$ des Espagnols arrivés en France au cours des cinq années précédentes étaient diplômés du supérieur, chiffres nettement plus élevés que pour les migrants des autres pays d'Europe ( $48 \%$ pour les Britanniques et $43 \%$ pour les Belges), et que pour les migrants non européens $(36 \%)^{12}$. Il faut voir là une conséquence de la forte marginalisation des jeunes sur le marché du travail qui, en Europe du Sud, touche également les diplômés. Ainsi, alors qu'en France ou au Royaume-Uni, le chômage des jeunes décroît fortement en fonction du degré de qualification, en Italie, la tendance est presque inverse : parmi les 20-29 ans, le taux de chômage est plus élevé chez les jeunes de niveau « bac +5 » que chez ceux « bac $+2 »^{13}$. Pays où l'accès à l'enseignement supérieur a été massif mais récent, et dont la croissance économique a largement reposé sur de petites entreprises familiales et une main-d'œuvre peu qualifiée dans les années d'après-guerre, l'Italie est aujourd'hui confrontée à une surqualification structurelle des jeunes générations, parfois poussées à émigrer pour trouver des emplois salariés conformes à leur niveau de diplôme. 
La crise économique a encore accentué cette émigration hautement qualifiée dans la péninsule : la part des Italiens diplômés du supérieur résidant à l'étranger quatre ans après avoir obtenu leur diplôme a presque doublé entre 2011 et 2015, pour atteindre $4,7 \%$ de leur cohorte ${ }^{14}$. Parmi ces derniers, $40 \%$ se sont installés en Europe occidentale (France, Allemagne, Suisse, Pays-Bas, Belgique, Irlande et Royaume-Uni) ; et seule une minorité (20 \%) a franchi les frontières du vieux continent. Les très bons étudiants sont légèrement surreprésentés parmi les diplômés expatriés, tout comme ceux ayant déjà eu des premières expériences à l'étranger et ceux originaires des régions riches du Nord, comme si l'émigration ne venait plus compenser - comme auparavant - mais plutôt renforcer les inégalités sociales et territoriales de la péninsule. Les données de l'Insee sur l'âge des migrants italiens en France confirment ces tendances. Parmi les migrants arrivés récemment, les Italiens se distinguent par le poids très élevé de la classe 25-44 ans, soit des actifs en première phase de carrière. En 2011, ces derniers représentaient plus de $52 \%$ des Italiens arrivés en France au cours des cinq années précédentes, contre seulement $26 \%$ des Britanniques ou $37 \%$ des Belges ${ }^{15}$. La nouvelle migration italienne en France, largement composée de jeunes actifs diplômés, se différencie ainsi assez nettement de celle en provenance d'Europe du Nord-Ouest où dominent les retraités et les cadres expatriés en fin de carrière, et également des migrations d'Europe de l'Est ou du Portugal, composées principalement de jeunes adultes, mais peu qualifiés ${ }^{16}$.

\section{La résurgence d'une « migration de métiers »}

Comment ces jeunes adultes qualifiés s'intègrentils sur le marché du travail français? Ici, les données manquent à l'échelle nationale, mais notre enquête par questionnaire permet d'en savoir un peu plus pour le cas parisien. Parmi les enquêtés, à savoir des Italiens arrivés à Paris depuis plus de trois mois, on trouve une grande majorité de cadres supérieurs (51 \% des actifs) et de professions intermédiaires (24\%), tandis que les employés et les ouvriers sont nettement sous-représentés (1 et $2 \%$ ). Leurs métiers se rattachent à des secteurs fortement implantés dans le centre de Paris et marqués traditionnellement par une importante présence italienne : certains peu qualifiés comme le commerce-tourisme-restauration (16\% des actifs), mais d'autres beaucoup plus comme la communicationinformation (7 \%), l'art, la $25 \%$ des Italiens actifs enquêtés en 2015 étaient également titulaires d'un contrat précaire (stage ou $(\mathrm{CDD})$. On est donc loin de l'image de cadres de la mondialisation circulant facilement entre métropoles internationales, volontiers mise en avant par certaines études sur les migrants très qualifiés.

mode et la culture ( $9 \%$ ) et

- surtout - l'enseignement et la recherche (26\%). Ce dernier secteur est marqué par une présence italienne particulièrement notable : avec 313 chercheurs, soit $19 \%$ des chercheurs étrangers, les Italiens sont le premier contingent de chercheurs étrangers titulaires au $\mathrm{CNRS}^{17}$. Le secteur de la finance, pourtant surreprésenté à Paris, n'attire en revanche qu'une faible proportion des nouveaux migrants italiens (3\%). Ces données sur les professions obligent à nuancer un peu la discontinuité entre anciennes et nouvelles vagues migratoires. Les activités des jeunes Italiens à Paris s'inscrivent dans la tradition d'une "migration de métiers" entre la France et l'Italie, où dominent les professions très qualifiées de l'art et de la culture, et qui n'a jamais cessé depuis la Renaissance, même si elle a été un temps occultée par l'importance de la migration de masse.

Cette forte contribution à l'économie de la connaissance de la métropole parisienne ne se fait cependant pas sans difficultés. Malgré leur qualification, 
les nouveaux migrants italiens peuvent traverser des périodes de précarité résidentielle et professionnelle dans la capitale. En 2015, seule une petite minorité d'entre eux gagnent plus de 5000 euros nets par mois et peuvent se rattacher à une «élite migrante ", tandis que la moitié gagne moins de 2000 euros, et près d'un quart touche même moins de 1200 euros par mois. $25 \%$ des Italiens actifs enquêtés en 2015 étaient également titulaires d'un contrat précaire (stage ou CDD). On est donc loin de l'image de cadres de la mondialisation circulant facilement entre métropoles internationales, volontiers mise en avant par certaines études sur les migrants très qualifiés. Les nouveaux migrants italiens à Paris se rattachent plutôt à une classe moyenne diplômée poussée à la migration pour maintenir son statut dans un contexte de déclassement et de précarisation à l'ère de l'éducation de masse. Le travail ne joue d'ailleurs qu'un rôle partiel dans les motivations de leur venue en France.

\section{Au-delà du travail, une génération en quête d'émancipation}

Lorsqu'on les interroge sur les motivations de leur migration, les jeunes migrants italiens à Paris insistent moins sur le travail proprement dit - beaucoup avaient déjà un emploi en Italie - que sur le niveau de responsabilité professionnelle et le degré de reconnaissance que ce dernier leur apporte en France, où ils s'estiment recrutés à leur juste niveau de compétence.

"On est tous conscients quion fait des choses ici qu'on ne peut pas faire en Italie. Tout simplement, on nous a donné des responsabilités ici qu'on n'aurait jamais eues en Italie. [...] Je n'aurais jamais pu faire un travail éditorial en Italie à 30 ans, impossible, je n'aurais jamais été chargée de mission pour un directeur d'une grande ONG comme WWF en Italie. Tout simplement parce que la culture italienne ne te permet pas de prendre des responsabilités à cet âge-là. On ne te considère pas, on ne prend pas le risque en fait, alors que les Français oui. » (Alessandra, cadre dans une ONG, 31 ans).

"Paris, c'est une super ville. Y a pas le manque de méritocratie comme en Italie. Les talents, les qualités, sont reconnus [...] ici, si on a un CV derrière, il y a la possibilité. En Italie, si t'es pas un "fils de", tu peux pas. » (Adriano, artiste, 38 ans).

Plus que la crise financière et économique, c'est donc une crise structurelle de la société italienne qui est au centre de cette nouvelle vague migratoire, celle de la marginalisation des jeunes dans les sociétés d'Europe du Sud. Ces dernières sont en effet marquées par un "syndrome du retard $^{18}$ » : retard de l'accès à un emploi stable et légal, de l'accès à l'indépendance résidentielle, souvent après 35 ans, retard de la première naissance, etc. La migration est ainsi vue comme un moyen de raccourcir cette longue phase de transition vers l'indépendance personnelle : " [En Italie], à 40 ans on commence à se dire que oui, on peut éventuellement commencer à grandir. Mais on reste soumis à une espèce d'infantilisation très longue. Et c'est aussi la conséquence du fait que les gens travaillent jusqu'à un âge très poussé. [...] On le voit aussi dans les entreprises, dans la classe politique : ce sont des gens qui ont 80 ans, ils sont toujours là. Donc il n'y a juste pas de place à un moment donné. La personne jeune qui arrive se range derrière, la suivante également, et tu t'entasses jusqu'à 40 ans [...]. Moi, j'ai commencé aussi à le voir par ma scur qui a commencé avant moi à chercher des expériences et qui s'est vite rendu compte qu'il fallait aller à l'étranger si elle voulait vraiment faire un truc concret tout de suite. » (Alessandra, cadre dans une ONG, 31 ans).

Ce manque de reconnaissance et d'opportunités suscite un « sentiment générationnel » qui se renforce dans la migration. Les enquêtés racontent leur parcours individuel comme une expérience collective et générationnelle, passant fréquemment du « je » au «nous » dans les entretiens, et se comparant souvent à la génération de leurs parents. Ils se décrivent comme une "généra- 
tion sacrifiée », ou "génération personne ${ }^{19}$ », rejetée par une Italie " gérontocratique » incapable de répondre au défi du renouvellement générationnel. "Une chose que je partage beaucoup avec mes compatriotes ici, c'est l'angoisse sur le destin de l'Italie. Je me rends compte quon en parle souvent. Maintenant, je regarde chaque jour le journal Repubblica sur Internet, comme tous mes amis. On regarde tout le temps quoi, le destin maudit de l'Italie. [...] Facebook c'est le moyen le plus intéressant pour partager une chanson triste sur l'Italie, la dernière bêtise de Berlusconi [...]. En fait, $90 \%$ de mes amis qui ont été de gauche comme moi ont voté Grillo. Tous les amis de mon âge de 33 ans environ, à Paris, ont voté Grillo. C'est la génération qui change. Il y a rien à faire, il faut attendre que ceux qui ont 70, 80 ans meurent. » (Giovanni, travailleur associatif, 33 ans).

Ces paroles reflètent l'omniprésence du thème de la «fracture générationnelle » en Italie, que ce soit dans les médias et le débat politique - l'ascension du mouvement Cinq étoiles ${ }^{20}$ a largement reposé sur le vote des jeunes et les promesses de renouvellement générationnel -, ou dans la recherche académique, qui insiste sur les nouvelles divisions de la société italienne au-delà de la traditionnelle structure de classes, comme les inégalités géographiques, de genre et de génération ${ }^{21}$. Ce souhait d'émancipation et de reconnaissance générationnelle, qui agit comme un moteur de la migration, n'est toutefois pas synonyme de rupture avec les parents. Les échanges avec l'Italie restent nombreux, favorisés par la proximité et la facilité des moyens de communication. Les solidarités familiales se recomposent à l'échelle transnationale: grands-parents italiens venant aider leurs enfants pendant les vacances scolaires, adultes rendant visite à leurs parents vieillissants au pays, familles recomposées des deux côtés des Alpes, envois d'argent dans les deux directions.

\section{L'attraction de Paris et des métropoles}

Ce rôle du désir d'émancipation dans la migration se ressent aussi dans les destinations des nouveaux migrants italiens en France. Ces dernières sont largement orientées vers les grandes métropoles et Paris, et non vers les zones traditionnelles de l'immigration italienne comme les campagnes du Sud ou les bassins miniers du Nord-Est. Dans les entretiens, les enquêtés insistent sur l'univers d'opportunités au sens large - professionnelles, sociales, culturelles - que leur ouvre la métropole parisienne, en permettant une réalisation personnelle impossible dans leur pays d'origine: "Nous avons vécu pendant deux mois à Orléans, en province. Et j'ai pensé : vivre en France, d'accord, mais dans ce cas il faut que ce soit à Paris, et rien d'autre! " (Isabella, 30 ans, universitaire). Cette attraction pour les grandes villes est un trait commun de nombre de nos interlocuteurs, qui préfèrent parfois s'installer dans des collocations peu confortables et coûteuses plutôt que d'habiter dans des périphéries trop distantes du centre. Cette métropolisation des flux se retrouve d'ailleurs dans d'autres lieux de l'émigration italienne, notamment

Les solidarités familiales se recomposent à l'échelle transnationale: grands-parents italiens venant aider leurs enfants pendant les vacances scolaires, adultes rendant visite à leurs parents vieillissants au pays, familles recomposées des deux côtés des Alpes, envois d'argent dans les deux directions. au Royaume-Uni ${ }^{22}$.

Elle s'exprime aussi à l'échelle intra-urbaine, comme le montre la carte de la répartition résidentielle des Italiens ayant répondu au questionnaire (voir carte) ${ }^{23}$. Dans l'agglomération parisienne, ces derniers délaissent nettement la banlieue et les 
zones traditionnelles d'implantation italienne (de Montreuil au Val-de-Marne) pour se concentrer dans la partie centrale. Lorsqu'ils sont présents audelà du périphérique, c'est aux abords immédiats de Paris, dans la première couronne bien desservie par le métro, et qui présente de plus en plus les caractéristiques de Paris intra-muros par ses activités (diffusion des bureaux et des grands équipements culturels) et sa composition sociale (extension du front de gentrification). Cette attraction pour la centralité parisienne est symptomatique d'une migration de jeunes adultes en quête d'opportunités et privilégiant des quartiers denses, socialement, culturellement et fonctionnellement diversifiés. On retrouve la même situation à Londres : parmi les Italiens de la capitale britannique, « anciens » et « nouveaux » migrants se différencient clairement tant en termes de localisation résidentielle que de lieux de sociabilités ${ }^{24}$.

Dans Paris intra-muros, ces nouveaux migrants italiens se distinguent par une distribution résidentielle très diffuse et recouvrant un éventail large de quartiers aux caractéristiques sociales variées. La dichotomie Nord-Est/Sud-Ouest, nette sur la carte des Italiens au recensement de $1968^{25}$, s'est fortement estompée. Les Italiens sont aujourd'hui présents dans tout le quart Nord-Est de Paris, mais également dans les XVe et XIVe arrondissements (secteurs de Montparnasse et d'Alésia). La carte actuelle ne fait pas ressortir de concentration nette, ou de "quartier italien ». Certes, Paris n'a jamais compté de véritable Little Italy, aussi structurée que les petites Italies des Amériques, mais dans les années 1950-1960 les migrants italiens tendaient tout de même à se regrouper en blocs et en rues contiguës, dessinant parfois des centralités ethniques bien visibles dans l'espace urbain ${ }^{26}$. Aujourd'hui, le degré de dispersion semble si important que de telles micro-centrali- tés n'apparaissent plus. Le traditionnel modèle de l'assimilation spatiale semble ne pas fonctionner ici. Contrairement à leurs prédécesseurs qui se regroupaient par origine régionale pour faciliter leur arrivée en France avant de se disperser en banlieue avec la réussite des générations suivantes, les migrants italiens actuels, plus qualifiés, se dispersent d'emblée dans l'espace urbain, selon un modèle d'« hétérolocalisme » déjà observé dans d'autres métropoles européennes ${ }^{27}$.

\section{Conclusion}

L'immigration italienne récente se distingue donc nettement de l'immigration de masse des années 1860-1970 par des effectifs plus faibles, un niveau de qualification nettement plus élevé, et des parcours plus clairement métropolitains. Les migrants récents ne sont pas venus en France simplement pour trouver un travail, mais mettent explicitement en avant des motivations existentielles et affectives, un désir de reconnaissance méritocratique, et plus largement un besoin de fuir un pays qu'ils jugent en situation de crise structurelle multiforme, à la fois sociale et morale, et qui bloque les perspectives de promotion sociale pour toute une génération. La dimension générationnelle constitue ainsi une caractéristique forte de cette émigration. Elle sert à se différencier des « anciens » immigrés pour de jeunes actifs qui évoquent volontiers la flexibilité géographique de leurs projets professionnels et leur capacité à se projeter, sinon à l'échelle du monde, du moins à celle de l'Europe, dont ils utilisent fréquemment les dispositifs institutionnels d'incitation à la mobilité (Erasmus notamment). Les entretiens font au final apparaître un très clair «sentiment générationnel » qui sous-tend une critique véhémente adressée à l'en-

22. Iside Gjergji, op. cit. 23. La question était ainsi formulée : "Quelle est la station (métro ou RER) la plus proche de votre domicile?" 24. Giuseppe Scotto, "From Emigrants" to "Italians": what is new in Italian migration to London ", in Modern Italy, vol. 20, n², 2015, pp. 153-155. 25. Marie-Claude Blanc-Chaléard, Pierre Milza, "Les Italiens à Paris depuis 1945 ", in Antoine Marès, Pierre Milza (dir.), Le Paris des étrangers depuis 1945, Paris, Publications de la Sorbonne, 1995, pp. 33-53. 26. Judith Rainhorn, Paris, New York, des migrants italiens, années 1880-1930, Paris, CNRS éd., 2005. 27. Wilbur Zelinsky, Barrett A. Lee, "Heterolocalism: an alternative model of the sociospatial behaviour of immigrant ethnic communities ", in International Journal of Population Geography, vol. 4, $\mathrm{n}^{\circ} 4,1998$, pp. 281-298. 


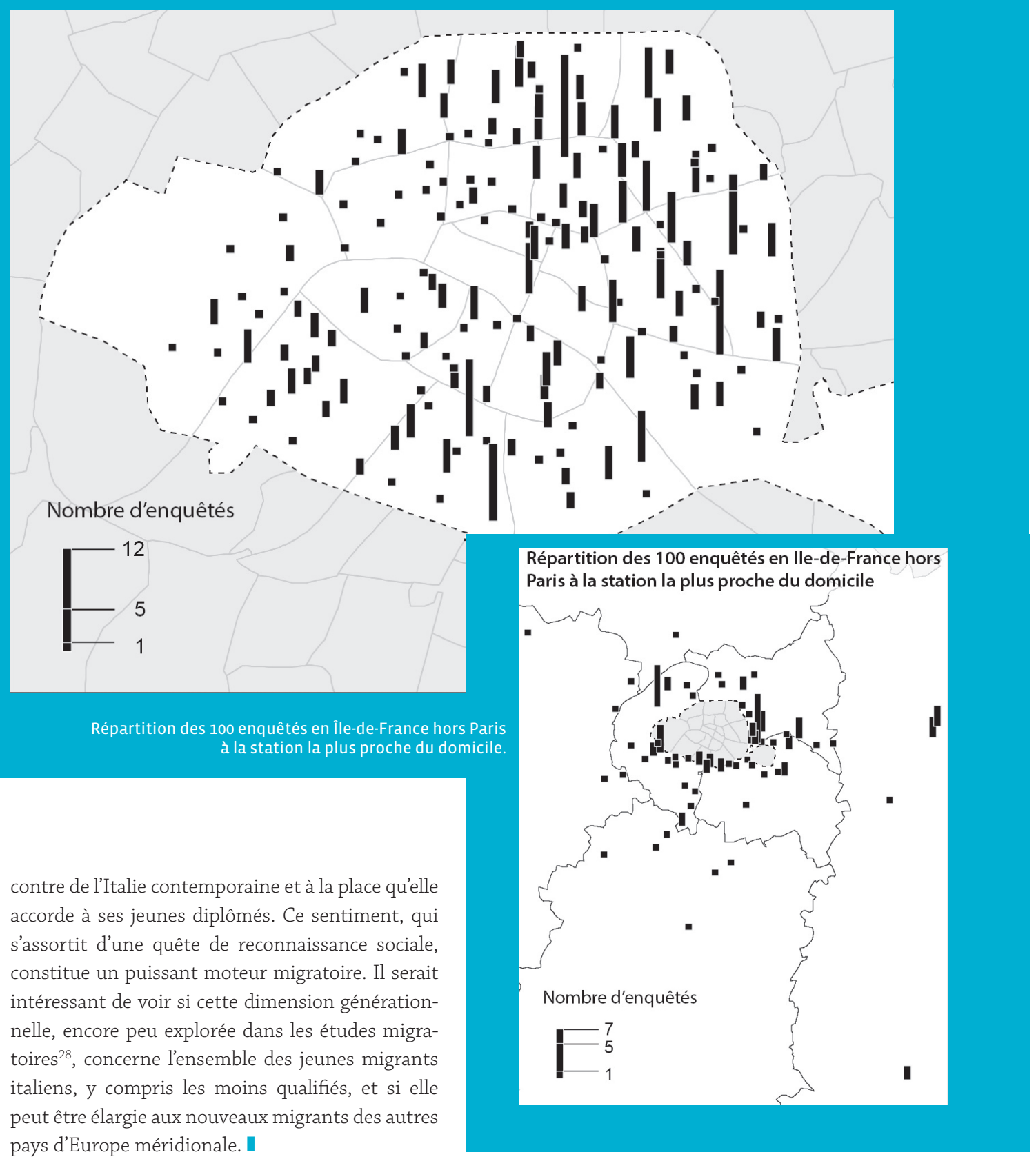

